\title{
On the Ricci symmetry of almost Kenmotsu manifolds
}

\author{
Dibakar Dey
}

\begin{abstract}
In the present paper, we characterize Ricci symmetric almost Kenmotsu manifolds under several constraints and proved that they are Einstein manifolds. As a consequence, we obtain several corollaries. Finally, an illustrative example is presented to verify our results.
\end{abstract}

Keywords. Almost Kenmotsu manifolds, Ricci symmetric manifolds, Einstein manifold, Sectional curvature

\section{Introduction}

As an extension of the well-known Kenmotsu manifolds (see [11]) and an analogy of almost Hermitian manifolds for manifolds of odd dimension, almost Kenmotsu manifolds defined in [9] are becoming an important research object in differential geometry of almost contact metric manifolds. Almost Kenmotsu manifolds satisfying the $(k, \mu)$ and $(k, \mu)^{\prime}$-nullity conditions were firstly introduced and studied by Dileo and Pastore [7], where $k$ and $\mu$ both are constants. As a special case of the $(k, \mu)$ and $(k, \mu)^{\prime}$-nullity conditions, $k$-nullity condition defined on almost Kenmotsu manifolds by Pastore and Saltarelli [13]. Later, Pastore and Saltarelli in [14] extended the above three nullity conditions to the corresponding generalized nullity conditions for which both $k$ and $\mu$ are assumed to be smooth functions.

It is well known that symmetric spaces play an important role in differential geometry. The study of locally symmetric Riemannian spaces was initiated in the late twenties by Cartan [3], who, in particular, obtained a classification of those spaces. Let $\left(M^{n}, g\right)$ be a Riemannian manifold of dimension $n$, i.e., a manifold $M^{n}$ with the Riemannian metric $g$ and let $\nabla$ be the LeviCivita connection of $\left(M^{n}, g\right)$. A Riemannian manifold is called locally symmetric [3] if $\nabla R=0$, where $R$ is the Riemannian curvature tensor of $\left(M^{n}, g\right)$. This condition of local symmetry is equivalent to the fact that at every point $p \in M^{n}$, the local geodesic symmetry $F(p)$ is an isometry [12]. A Riemannian manifold is called Ricci symmetric if $\nabla S=0$, where $S$ is the Ricci tensor of $\left(M^{n}, g\right)$. The class of locally symmetric Riemannian manifolds is very natural generalization of the class of manifolds of constant curvature. During the last five decades the notion of locally symmetric manifolds have been weakened by many authors in several ways.

In a recent paper, Dileo and Pastore [6] studied locally symmetric almost Kenmotsu manifolds under the condition that the Lie derivative of the structure tensor $\phi$ with respect to the Reeb

Received date: June 27, 2020; Published online: April 04, 2021.

2010 Mathematics Subject Classification. 53D15, 53C25.

Corresponding author: Dibakar Dey. 
vector field vanishes. Also Wang and Liu [16] studied classification problems on locally symmetric almost Kenmotsu manifolds and proved that a three dimensional locally symmetric almost Kenmotsu manifold is either hyperbolic space $\mathbb{H}^{3}(-1)$ or the Riemannian product $\mathbb{H}^{2}(-4) \times \mathbb{R}$. Deshmukh, De and Zhao [8] studied Ricci semisymmetric almost Kenmotsu manifolds with nullity distributions. In [15], Sharma studied Ricci symmetric contact metric manifolds under several constraints and conformally flat contact metric manifolds assuming certain curvature condition. Further, Ghosh and Majhi [10] studied Ricci semisymmetric almost Kenmotsu manifolds with conformal Reeb foliation.

Motivated by the above studies, in the present paper we consider Ricci symmetric almost Kenmotsu manifolds under several constraints and proved that they are Einstein manifolds. Finally, we have verified our results with an example.

\section{Almost Kenmotsu Manifolds}

An almost contact structure on a $(2 n+1)$-dimensional smooth manifold $M^{2 n+1}$ is a triplet $(\phi, \xi, \eta)$, where $\phi$ is a $(1,1)$-tensor, $\xi$ is a global vector field and $\eta$ is a 1 -form satisfying (see $[1,2])$

$$
\phi^{2}=-I+\eta \otimes \xi, \quad \eta(\xi)=1,
$$

where $I$ denote the identity endomorphism. Here also holds $\phi \xi=0$ and $\eta \circ \phi=0$; both can be derived from (2.1) easily.

If a manifold $M^{2 n+1}$ with a $(\phi, \xi, \eta)$-structure admits a Riemannian metric $g$ such that

$$
g(\phi X, \phi Y)=g(X, Y)-\eta(X) \eta(Y)
$$

for any vector fields $X, Y$ in $T\left(M^{2 n+1}\right)$, then $M^{2 n+1}$ is said to be an almost contact metric manifold. The fundamental 2 -form $\Phi$ on an almost contact metric manifold is defined by

$$
\Phi(X, Y)=g(X, \phi Y)
$$

for any $X, Y$ in $T\left(M^{2 n+1}\right)$. The condition for an almost contact metric manifold being normal is equivalent to vanishing of the $(1,2)$-type torsion tensor $N_{\phi}$, defined by

$$
N_{\phi}=[\phi, \phi]+2 d \eta \otimes \xi,
$$

where $[\phi, \phi]$ is the Nijenhuis tensor of $\phi[1]$. Recently in $[5,6,7]$, almost contact metric manifold such that $\eta$ is closed and $d \Phi=2 \eta \wedge \Phi$ are studied and they are called almost Kenmotsu manifolds. Obviously, a normal almost Kenmotsu manifold is a Kenmotsu manifold. Also Kenmotsu manifolds can be characterized by

$$
\left(\nabla_{X} \phi\right) Y=g(\phi X, Y) \xi-\eta(Y) \phi X,
$$

for any vector fields $X, Y$ in $T\left(M^{2 n+1}\right)$.

Let $M^{2 n+1}$ be an almost Kenmotsu manifold. We denote by $h=\frac{1}{2} £_{\xi} \phi$ and $l=R(\cdot, \xi) \xi$ on $M^{2 n+1}$. The tensor fields $l$ and $h$ are symmetric operators and satisfy the following relations [13]:

$$
h \xi=0, l \xi=0, \operatorname{tr}(h)=0, \operatorname{tr}(h \phi)=0, h \phi+\phi h=0,
$$

We also have the following formulas (see $[5,7,11]$ )

$$
\nabla_{X} \xi=X-\eta(X) \xi-\phi h X
$$




$$
\begin{gathered}
\phi l \phi-l X=2\left(h^{2}-\phi^{2}\right), \\
\operatorname{tr}(l)=S(\xi, \xi)=g(Q \xi, \xi)=-2 n-\operatorname{tr}\left(h^{2}\right), \\
R(X, Y) \xi=\eta(X)(Y-\phi h Y)-\eta(Y)(X-\phi h X)+\left(\nabla_{Y} \phi h\right) X-\left(\nabla_{X} \phi h\right) Y, \\
\nabla_{\xi} h=-\phi-2 h-\phi h^{2}-\phi l
\end{gathered}
$$

for any $X, Y$ in $T\left(M^{2 n+1}\right)$. Let $X \in \mathcal{D}$ be an eigenvector of $h$ corresponding to the eigenvalue $\lambda$. Then $-\lambda$ is also an eigenvalue of $h$. We denote the eigenspaces associated with $h$ by $[\lambda]$ and $[-\lambda]$ corresponding to the non-zero eigenvalues $\lambda$ and $-\lambda$ respectively. We refer the reader to go through the references $[4,6,8]$ for further details on almost Kenmotsu manifolds.

\section{Ricci Symmetric Almost Kenmotsu Manifolds}

In this section, we consider Ricci symmetric almost Kenmotsu manifolds under several conditions.

Lemma 3.1. In an almost Kenmotsu manifold the following conditions holds

(i) $\operatorname{tr}\left(h^{2} Q\right)=\operatorname{tr}\left(Q h^{2}\right)$

(ii) $\operatorname{tr}(\phi h Q)=\operatorname{tr}(Q \phi h)$

(iii) $\operatorname{tr}\left(\phi\left(\nabla_{\xi} h\right) Q\right)=\operatorname{tr}\left(Q \phi\left(\nabla_{\xi} h\right)\right)$

Proof. (i) $\operatorname{tr}\left(h^{2} Q\right)=g\left(h^{2} Q e_{i}, e_{i}\right)=g\left(Q e_{i}, h^{2} e_{i}\right)=g\left(e_{i}, Q h^{2} e_{i}\right)=\operatorname{tr}\left(Q h^{2}\right)$, where the symmetry of $Q$ and $h$ are used.

(ii) $\operatorname{tr}(\phi h Q)=g\left(\phi h Q e_{i}, e_{i}\right)=-g\left(h Q e_{i}, \phi e_{i}\right)=-g\left(Q e_{i}, h \phi e_{i}\right)=g\left(Q e_{i}, \phi h e_{i}\right)=g\left(e_{i}, Q \phi h e_{i}\right)=$ $\operatorname{tr}(Q \phi h)$, where the skew symmetry of $\phi$, the symmetry of $Q$ and $h$ and the fact $h \phi+\phi h=0$ are used.

(iii) From (2.7), we have

$$
\phi\left(\nabla_{\xi} h\right) X=h^{2} X+X-\eta(X) \xi-2 \phi h X+l X .
$$

Now, using the foregoing equation, we infer that

$$
\begin{aligned}
\operatorname{tr}\left(\phi\left(\nabla_{\xi} h\right) Q\right) & =g\left(h^{2} Q e_{i}, e_{i}\right)+g\left(Q e_{i}, e_{i}\right)-g\left(\eta\left(Q e_{i}\right) \xi, e_{i}\right)-2 g\left(\phi h Q e_{i}, e_{i}\right)+g\left(l Q e_{i}, e_{i}\right) \\
& =\operatorname{tr}\left(h^{2} Q\right)+r-S(\xi, \xi)-2 \operatorname{tr}(\phi h Q)+\operatorname{tr}(l Q) .
\end{aligned}
$$

Again,

$$
\begin{aligned}
\operatorname{tr}\left(Q \phi\left(\nabla_{\xi} h\right)\right) & =g\left(Q h^{2} e_{i}, e_{i}\right)+g\left(Q e_{i}, e_{i}\right)-g\left(\xi, e_{i}\right) \cdot g\left(Q \xi, e_{i}\right)-2 g\left(Q \phi h e_{i}, e_{i}\right)+g\left(Q l e_{i}, e_{i}\right) \\
& =\operatorname{tr}\left(Q h^{2}\right)+r-S(\xi, \xi)-2 \operatorname{tr}(Q \phi h)+g\left(e_{i}, l Q e_{i}\right) \\
& =\operatorname{tr}\left(Q h^{2}\right)+r-S(\xi, \xi)-2 \operatorname{tr}(Q \phi h)+\operatorname{tr}(l Q) .
\end{aligned}
$$

Hence, using (i) and (ii), we can see that $\operatorname{tr}\left(\phi\left(\nabla_{\xi} h\right) Q\right)=\operatorname{tr}\left(Q \phi\left(\nabla_{\xi} h\right)\right)$.

Theorem 3.1. If an almost Kenmotsu manifold $M^{2 n+1}$ is Ricci symmetric, then 
(i) $\operatorname{tr}(Q l)=\|Q \xi\|^{2}$

(ii) the scalar curvature $r=-2 n-\operatorname{tr}\left(Q\left(h^{2}-2 \phi h-\phi\left(\nabla_{\xi} h\right)\right)+h^{2}\right)-\|Q \xi\|^{2}$

(iii) the sectional curvature $K(\xi, Q \xi)=0$ if $Q \xi$ is not collinear with $\xi$.

Proof. Let the Ricci tensor of an almost Kenmotsu manifold $M^{2 n+1}$ is parallel, i.e., $\nabla S=0$ holds on $M^{2 n+1}$, where $S$ is the Ricci tensor. Then we have

$$
S(R(W, X) Y, Z)+S(R(W, X) Z, Y)=0,
$$

which implies

$$
g(R(W, X) Y, Q Z)+g(R(W, X) Z, Q Y)=0,
$$

where $Q$ is the Ricci operator defined by $g(Q X, Y)=S(X, Y)$. Now the foregoing equation can be written as

$$
g(R(Y, Q Z) W, X)+g(R(Z, Q Y) W, X)=0 .
$$

Putting $Y=W=\xi$ in (3.1), we get

$$
g(R(\xi, Q Z) \xi, X)+g(R(Z, Q \xi) \xi, X)=0 .
$$

Now substituting $Z=\xi$ and $X=Q \xi$ in (3.2) we obtain $g(R(\xi, Q \xi) \xi, Q \xi)=0$ and therefore, $K(\xi, Q \xi)=0$ if $Q \xi$ is not collinear with $\xi$. This proves (iii).

From (2.7), we get

$$
\phi\left(\nabla_{\xi} h\right) X=-\phi^{2} X-2 \phi h X-\phi^{2} h^{2} X-\phi^{2} l X,
$$

which implies

$$
R(\xi, X) \xi=h^{2} X+X-\eta(X) \xi-2 \phi h X-\phi\left(\nabla_{\xi} h\right) X .
$$

Making use of (3.3) in (3.2), we have

$$
g\left(h^{2} Q Z+Q Z-\eta(Q Z) \xi-2 \phi h Q Z-\phi\left(\nabla_{\xi} h\right) Q Z, X\right)+g(R(Z, Q \xi) \xi, X)=0 .
$$

Putting $Z=X=e_{i}$ in (3.4), where $\left\{e_{i}\right\}, i=1,2, \ldots,(2 n+1)$ is an orthonormal basis of the tangent space at each point of the manifold and taking summation over $i$, we infer that

$$
\operatorname{tr}\left(h^{2} Q\right)+r-S(\xi, \xi)-2 \operatorname{tr}(\phi h Q)-\operatorname{tr}\left(\phi\left(\nabla_{\xi} h\right) Q\right)+\|Q \xi\|^{2}=0,
$$

where $r$ is the scalar curvature. Using (2.5) and Lemma 3.1, we have from (3.5)

$$
r=-2 n-\operatorname{tr}\left(Q\left(h^{2}-2 \phi h-\phi\left(\nabla_{\xi} h\right)\right)+h^{2}\right)-\|Q \xi\|^{2} .
$$

This proves (ii).

Now operating $Q$ on (3.3) and then tracing the resulting equation gives

$$
-\operatorname{tr}(Q l)=\operatorname{tr}\left(Q h^{2}\right)+r-S(\xi, \xi)-2 \operatorname{tr}(Q \phi h)-\operatorname{tr}\left(Q \phi\left(\nabla_{\xi} h\right)\right) .
$$

Therefore, upon using Lemma 3.1, (3.5) and (3.7) together implies

$$
\operatorname{tr}(Q l)=\|Q \xi\|^{2}
$$

which proves (i). 
Corollary 3.2. A Ricci symmetric almost Kenmotsu manifold $M^{2 n+1}$ with zero $\xi$-sectional curvature (i.e., $l=0$ ) is Ricci flat.

Proof. If $l=0$, then from (i) of the above theorem, we have $Q \xi=0$.

Differentiating it along $\phi X$, where $X \perp \xi$, we get $Q(\phi X)=Q(h X)$. As $X \perp \xi$, replacing $X$ by $\phi X$ in the previous equation, we have $Q X=Q \phi h X$. Again substituting $h X=\lambda X, X \perp \xi$ in the foregoing equation, we obtain $Q X=\lambda Q \phi X$. Now replacing $X$ by $\phi X$ as $X \perp \xi$ in the last equation yields $Q \phi X=-\lambda Q X$. Now the last two equations together implies $\left(1+\lambda^{2}\right) Q X=0$. Since $h$ is symmetric, $\left(1+\lambda^{2}\right) \neq 0$ and therefore, $Q X=0$ for all vector field $X \perp \xi$. Thus $Q X=0$ for all vector field $X$ on $M^{2 n+1}$, which implies that the manifold is Ricci flat. This completes the proof.

Theorem 3.3. If a Ricci symmetric almost Kenmotsu manifold $M^{2 n+1}$ satisfies $Q \xi=f \xi$ for some smooth function $f$ on $M^{2 n+1}$, then $M^{2 n+1}$ is Einstein.

Proof. Since $Q \xi=f \xi$ holds on $M^{2 n+1}$, then $f=S(\xi, \xi)$. Taking covariant derivative of this along any vector field $X$, we have

$$
\begin{aligned}
X f & =2 S\left(\nabla_{X} \xi, \xi\right) \\
& =2 S(X-\eta(X) \xi-\phi h X, \xi) \\
& =2 S(X, \xi)-2 \eta(X) S(\xi, \xi) \\
& =0,
\end{aligned}
$$

which implies $f=$ constant.

Now, since $\nabla Q=0$, differentiating $Q \xi=f \xi$ along any vector field $X$, we obtain

$$
Q X-Q \phi h X=f X-f \phi h X .
$$

Let us consider $X \perp \xi$. Replacing $X$ by $\phi X$ in (3.9), we get

$$
Q \phi X-Q h X=f \phi X-f h X .
$$

Taking $h X=-\lambda X$ as $X \perp \xi$ in (3.10), we infer

$$
Q \phi X+\lambda Q X=f \phi X+f \lambda X .
$$

Again substituting $h X=\lambda X$ in (3.9) yields

$$
Q X-\lambda Q \phi X=f X-f \lambda \phi X .
$$

Multiplying the equation (3.11) by $\lambda$ and then adding with (3.12), we get

$$
\left(1+\lambda^{2}\right)(Q X-f X)=0 .
$$

Since, $h$ is symmetric, $1+\lambda^{2} \neq 0$. Hence, from (3.13), we have $Q X=f X$ for all $X \perp \xi$. Therefore, $Q X=f X$ for any vector field $X$ on $M^{2 n+1}$. This completes the proof.

Before proving our next theorem, we first state and prove the following Lemma:

Lemma 3.2. In an almost Kenmotsu manifold the condition $K(\xi, X)=K(\xi, \phi X)$ holds if and only if $\phi\left(\nabla_{\xi} h\right) X=2 h \phi X$. 
Proof. Using (3.3), we calculate the terms $K(\xi, X)$ and $K(\xi, \phi X)$ as follows:

$$
\begin{aligned}
K(\xi, X) & =-g(R(\xi, X) \xi, X) \\
& =-g(h X, h X)-1+2 g(\phi h X, X)+g\left(\phi\left(\nabla_{\xi} h\right) X, X\right)
\end{aligned}
$$

and

$$
K(\xi, \phi X)=-g(h X, h X)-1+2 g(h \phi X, X)+g\left(\left(\nabla_{\xi} h\right) \phi X, X\right) .
$$

Now from (2.4) and (2.7), we can easily obtain that $\left(\nabla_{\xi} h\right) \phi X=-\phi\left(\nabla_{\xi} h\right) X$. Now subtracting (3.15) and (3.15), we get

$$
K(\xi, X)-K(\xi, \phi X)=2 g\left(\phi\left(\nabla_{\xi} h\right) X-2 h \phi X, X\right) .
$$

Therefore, $K(\xi, X)=K(\xi, \phi X)$ holds if and only if $\phi\left(\nabla_{\xi} h\right) X=2 h \phi X$.

Theorem 3.4. If a Ricci symmetric almost Kenmotsu manifold $M^{2 n+1}$ satisfies $K(\xi, X)=$ $K(\xi, \phi X)$ and $K(\xi, X)>0$, then $M^{2 n+1}$ is an Einstein manifold.

Proof. Since the condition $K(\xi, X)=K(\xi, \phi X)$ is realized on $M^{2 n+1}$, we have $\phi\left(\nabla_{\xi} h\right) X=2 h \phi X$. From this, with the help of (2.7), we can easily obtain

$$
R(\xi, X) \xi=h^{2} X-\phi^{2} X .
$$

Now, for a Ricci symmetric almost Kenmotsu manifold, we have

$$
g(R(W, X) Y, Q Z)+g(R(W, X) Z, Q Y)=0 .
$$

Substituting $Y=Z=\xi$ in the foregoing equation and using (3.17), we obtain

$$
g\left(h^{2} X-\phi^{2} X, Q \xi\right)=0,
$$

which implies

$$
h^{2} Q \xi=S(\xi, \xi) \xi-Q \xi
$$

Now, suppose $V$ is an eigenvector of $h$ orthogonal to $\xi$ with eigenvalue $\lambda$, i.e., $h V=\lambda V$ and hence $h \phi V=-\lambda \phi V$. Taking inner product of (3.18) with $V$, we obtain

$$
\left(1+\lambda^{2}\right) g(Q \xi, V)=0 .
$$

Since $h$ is symmetric, $1+\lambda^{2} \neq 0$. This shows that for those eigenvectors $V$ with eigenvalue $\lambda$ (and hence for eigenvectors $\phi V$ with eigenvalue $-\lambda$ ), we get $Q \xi \perp V$ and $\phi V$. Also $V, \phi V \perp$ $\xi$. Therefore, $Q \xi \in L\{\xi\}$, where $L\{\xi\}$ is the linear span of $\xi$. Thus we have $Q \xi=f \xi$ for some function $f$. Now in a similar manner as in Theorem 3.3, we can obtain $Q X=f X$ for all $X \perp \xi$. Thus $Q=f I$ on $M^{2 n+1}$. This completes the proof.

If the sectional curvature $K(\xi, X)=c$ of an almost Kenmotsu manifold is independent of choice of $X$, then we can easily obtain the following:

$$
R(\xi, X) \xi=-c[X-\eta(X) \xi]
$$

which implies

$$
l X=c[X-\eta(X) \xi]
$$


Now from (2.4), we have

$$
\phi l \phi X-l X=2\left(h^{2}-\phi^{2}\right) X .
$$

Making use of (2.1) and (3.21) in the foregoing equation yields

$$
\phi l \phi X-c[X-\eta(X) \xi]=2\left[h^{2} X+X-\eta(X) \xi\right] .
$$

Putting $X=\phi X$ in (3.22) and using (2.1) and (3.21), we obtain

$$
-c \phi X=\left(h^{2} \phi X+\phi X\right) .
$$

Again substituting $X=\phi X$ in (3.23) and using (2.1), we get

$$
h^{2} X=(c+1)(-X+\eta(X) \xi) .
$$

Theorem 3.5. If a Ricci symmetric almost Kenmotsu manifold $M^{2 n+1}$ satisfies $K(\xi, X)=c$, where $c$ is a smooth function independent of choice of $X$ such that $c \neq 0,-2$, then $M^{2 n+1}$ is Einstein.

Proof. Let $M^{2 n+1}$ be an almost Kenmotsu manifold with the sectional curvature $K(\xi, X)=c$ $(\neq 0,-2$ and independent of choice of $X)$ such that $\nabla S=0$. Then it follows that

$$
S(R(W, X) Y, Z)+S(Y, R(W, X) Z)=0
$$

for any vector fields $X, Y, Z, W$ on $M^{2 n+1}$.

Substituting $W=Y=Z=\xi$ in (3.25) yields

$$
S(R(\xi, X) \xi, \xi)=0 .
$$

Using (3.20) in (3.26), we obtain

$$
-c S(X-\eta(X) \xi, \xi)=0 .
$$

As $c$ is non-zero, the above equation reduces to

$$
S(X, \xi)=S(\xi, \xi) g(X, \xi) .
$$

Differentiating (3.28) covariantly along $Y$, we get

$$
\begin{aligned}
S\left(\nabla_{Y} X, \xi\right)+S\left(X, \nabla_{Y} \xi\right)= & S(\xi, \xi) g\left(\nabla_{Y} X, \xi\right)+S(\xi, \xi) g\left(X, \nabla_{Y} \xi\right) \\
& +2 g(X, \xi) S\left(\nabla_{Y} \xi, \xi\right) .
\end{aligned}
$$

Replacing $X$ by $\nabla_{Y} X$ in (3.28), we obtain

$$
S\left(\nabla_{Y} X, \xi\right)=S(\xi, \xi) g\left(\nabla_{Y} X, \xi\right) .
$$

Using (3.30) in (3.29) yields

$$
S\left(X, \nabla_{Y} \xi\right)=S(\xi, \xi) g\left(X, \nabla_{Y} \xi\right)+2 g(X, \xi) S\left(\nabla_{Y} \xi, \xi\right) .
$$

Using (2.3), we get from (3.31)

$$
S(X, Y)-g(Y, \xi) S(X, \xi)-S(X, \phi h Y)
$$




$$
\begin{aligned}
=\quad & S(\xi, \xi)[g(X, Y)-g(Y, \xi) g(X, \xi)-g(X, \phi h Y)] \\
& +2 g(X, \xi)[S(Y, \xi)-g(Y, \xi) S(\xi, \xi)-S(\phi h Y, \xi)] .
\end{aligned}
$$

Substituting $Y=\phi Y$ in (3.32) and using (2.1)-(2.2), we have

$$
S(X, \phi Y)-S(X, h Y)=S(\xi, \xi)[g(X, \phi Y)-g(X, h Y)]
$$

Putting $Y=\phi Y$ in (3.33) and making use of (2.1), (2.2) and (3.28), we infer that

$$
S(X, Y)-S(X, \phi h Y)=S(\xi, \xi)[g(X, Y)-g(X, \phi h Y)] .
$$

Substituting $Y=h Y$ in the above equation, we have

$$
S(X, h Y)-S\left(X, \phi h^{2} Y\right)=S(\xi, \xi)\left[g(X, h Y)-g\left(X, \phi h^{2} Y\right)\right] .
$$

Putting the value of $h^{2} Y$ from (3.24) in (3.35), we get

$$
S(X, h Y)+(c+1) S(X, \phi Y)=S(\xi, \xi)[g(X, h Y)+(c+1) g(X, \phi Y)] .
$$

Now adding (3.33) and (3.36), we obtain

$$
S(X, \phi Y)=S(\xi, \xi) g(X, \phi Y),
$$

since $c \neq-2$ by hypothesis. Replacing $Y$ by $\phi Y$ in the foregoing equation and using (3.28), we have

$$
S(X, Y)=S(\xi, \xi) g(X, Y) .
$$

Tracing (3.20) yields $S(\xi, \xi)=2 n c$ and therefore, from (3.38), we get

$$
S(X, Y)=2 n c g(X, Y) .
$$

This completes the proof.

\section{Example of a 3-dimensional Einstein Almost Kenmotsu Manifold}

Consider $M^{3}=\left\{(x, y, z) \in \mathbb{R}^{3}: z \neq 0\right\}$. The vector fields

$$
E_{1}=z \frac{\partial}{\partial x}, \quad E_{2}=z \frac{\partial}{\partial y}, \quad E_{3}=-z \frac{\partial}{\partial z}
$$

are linearly independent at each point of $M^{3}$. Let $g$ be the Riemannian metric defined by

$$
\begin{aligned}
& g\left(E_{1}, E_{1}\right)=g\left(E_{2}, E_{2}\right)=g\left(E_{3}, E_{3}\right)=1, \\
& g\left(E_{1}, E_{2}\right)=g\left(E_{2}, E_{3}\right)=g\left(E_{3}, E_{1}\right)=0 .
\end{aligned}
$$

Let us consider $E_{3}=\xi$. The 1 -form $\eta$ is defined by $\eta(X)=g\left(X, E_{3}\right)$ for any vector field $X$ on $M^{3}$. The $(1,1)$-tensor field $\phi$ is defined by

$$
\phi\left(E_{1}\right)=-E_{2}, \quad \phi\left(E_{2}\right)=E_{1}, \quad \phi\left(E_{3}\right)=0 .
$$


Using linearity of $\phi$ and $g$ we have

$$
\eta(\xi)=1, \quad \phi^{2} X=-X+\eta(X) \xi \text { and } g(\phi X, \phi Y)=g(X, Y)-\eta(X) \eta(Y)
$$

for any vector field $X, Y$ on $M^{3}$. Now it is easy to see that

$$
\left[E_{1}, \xi\right]=E_{1}, \quad\left[E_{1}, E_{2}\right]=0 \text { and }\left[E_{2}, \xi\right]=0 .
$$

The well known Koszul's formula is given by

$$
\begin{aligned}
2 g\left(\nabla_{X} Y, Z\right)= & X g(Y, Z)+Y g(Z, X)-Z g(X, Y) \\
& -g(X,[Y, Z])-g(Y,[X, Z])+g(Z,[X, Y]) .
\end{aligned}
$$

Using the above Koszul's formula, we obtain the Levi-Civita connection $\nabla$ as follows:

$$
\begin{gathered}
\nabla_{E_{1}} E_{1}=-\xi, \quad \nabla_{E_{1}} E_{2}=0, \quad \nabla_{E_{1}} \xi=E_{1}, \\
\nabla_{E_{2}} E_{1}=0, \quad \nabla_{E_{2}} E_{2}=\xi, \quad \nabla_{E_{2}} \xi=E_{2}, \\
\nabla_{\xi} E_{1}=0, \quad \nabla_{\xi} E_{2}=0, \quad \nabla_{\xi} \xi=0 .
\end{gathered}
$$

In view of the above relations we have $\nabla_{X} \xi=X-\eta(X) \xi$ for any vector field $X$ on $M^{3}$. Thus $(\phi, \xi, \eta, g)$ is an almost contact metric structure such that $d \eta=0$ and $d \Phi=2 \eta \wedge \Phi$ and hence $M^{3}$ is an almost Kenmotsu manifold with $h=0$.

By the above relations, we can easily obtain the components of the curvature tensor $R$ as follows:

$$
\begin{array}{cll}
R\left(E_{1}, E_{2}\right) E_{1}=E_{2}, & R\left(E_{1}, E_{2}\right) E_{2}=-E_{1}, \quad R\left(E_{1}, E_{2}\right) \xi=0, \\
R\left(E_{2}, \xi\right) E_{1}=0, & R\left(E_{2}, \xi\right) E_{2}=\xi, & R\left(E_{2}, \xi\right) \xi=-E_{2}, \\
R\left(E_{1}, \xi\right) E_{1}=\xi, & R\left(E_{1}, \xi\right) E_{2}=0, & R\left(E_{1}, \xi\right) \xi=-E_{1} .
\end{array}
$$

With the help of the expressions of the curvature tensor, we see that

$$
R(X, Y) Z=-[g(Y, Z) X-g(X, Z) Y] .
$$

From above, we can easily obtain

$$
S(X, Y)=-2 g(X, Y) .
$$

Therefore, $M^{3}$ is an Einstein manifold.

Now, the $\xi$-sectional curvature is given by

$$
K\left(\xi, E_{1}\right)=K\left(\xi, E_{2}\right)=K\left(\xi, \phi E_{1}\right)=K\left(\xi, \phi E_{2}\right)=1 .
$$

Hence, Theorem 3.3, Theorem 3.4 and Theorem 3.5 are verified.

\section{Acknowledgments}

The author gratefully acknowledge the valuable comments of the anonymous referee. The author is thankful to the Council of Scientific and Industrial Research, India (File no: 09/028(1010)/2017EMR-1) for their assistance. 


\section{References}

[1] D. E. Blair, Contact manifold in Riemannian Geometry. Lecture Notes on Mathematics, Springer, Berlin, 509(1976).

[2] D. E. Blair, Riemannian Geometry on contact and symplectic manifolds, Progr. Math., Birkhäuser, Boston, 203(2010).

[3] E. Cartan, Sur une classes remarquable d'espaces de Riemannian, Bull. Soc. Math. France 54(1926), 214-264.

[4] U. C. De and D. Dey, Pseudo-symmetric structures on almost Kenmotsu manifolds with nullity distributions, Acta Comment. Univ. Tartu. Math. 23(2019), 13-24.

[5] G. Dileo and A. M. Pastore, Almost Kenmotsu manifolds and nullity distributions, J. Geom. 93(2009), 46-61.

[6] G. Dileo and A. M. Pastore, Almost Kenmotsu manifolds with a condition of $\eta$-parallelsim, Differential Geom. Appl. 27(2009), 671-679.

[7] G. Dileo and A. M. Pastore, Almost Kenmotsu manifolds and local symmetry, Bull. Belg. Math. Soc. Simon Stevin, 14(2007), 343-354.

[8] S. Deshmukh, U. C. De and P. Zhao, Ricci semisymmetric almost Kenmotsu manifolds with nullity distributions, Filomat 32(2018), 179-786.

[9] D. Janssens and L. Vanhecke, Almost contact structures and curvature tensors, Kodai Math. J. 4(1981), 1-27.

[10] G. Ghosh and P. Majhi, Certain results on almost Kenmotsu manifolds with conformal Reeb foliation, Commun. Korean Math. Soc. 33(2018), 261-272.

[11] K. Kenmotsu, A class of almost contact Riemannian manifolds, Tohoku Math. J. 24(1972), 93-103.

[12] B. O’Neill, Semi-Riemannian Geometry, Academic Press, Inc. NY 1983.

[13] A. M. Pastore and V. Saltarelli, Generalized nullity distribution on almost Kenmotsu manifolds, Int. Elec. J. Geom. 4(2011), 168-183.

[14] A. M. Pastore and V. Saltarelli, Almost Kenmotsu manifolds with conformal Reeb foliation, Bull. Belg. Math. Soc. Simon Stevin 21 (2012), 343-354.

[15] R. Sharma, On the curvature of contact metric manifolds, J. Geom. 53(1995), 179-190.

[16] Y. Wang, Three dimensional locally symmetric almost Kenmotsu manifolds, Ann. Polon. Math. 116(2016), 79-86.

Dibakar Dey Department of Pure Mathematics, University of Calcutta, 35, Ballygunge Circular Road, Kolkata - 700019, West Bengal, India.

E-mail: deydibakar3@gmail.com 